\title{
Comparative analysis of eukaryotic cell-free expression systems
}

\author{
Emily M. Hartsough ${ }^{1,2, \star}$, Pankti Shah ${ }^{3, \star}$, Andrew C. Larsen ${ }^{1, \star}$, \\ and John C. Chaput ${ }^{1,3}$

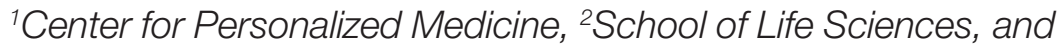 \\ ${ }^{3}$ Department of Chemistry and Biochemistry, Arizona State University, \\ Tempe, AZ. \\ *E.M.H., P.S., and A.C.L. contributed equally to this work.
}

BioTechniques 59:149-151 (September 2015) doi 10.2144/000114327

Keywords: recombinant protein synthesis; cell-free translation; translation enhancing elements

Supplementary material for this article is available at www.BioTechniques.com/article/114327.

Cell-free protein synthesis (CFPS) allows researchers to rapidly generate functional proteins independent of cell culture. Although advances in eukaryotic lysates have increased the amount of protein that can be produced, the nuances of different translation systems lead to variability in protein production. To help overcome this problem, we have compared the relative yield and template requirements for three commonly used commercial cell-free translation systems: wheat germ extract (WGE), rabbit reticulocyte lysate $(\mathrm{RRL})$, and HeLa cell lysate $(\mathrm{HCL})$. Our results provide a general guide for researchers interested in using cell-free translation to generate recombinant protein for biomedical applications.

Cell-free protein synthesis (CFPS) allows researchers to rapidly generate large numbers of proteins for a wide range of applications in biotechnology and biomedicine $(1,2)$. This method is especially attractive for proteins that are difficult to synthesize in traditional cell culture due to problems associated with cellular toxicity, low expression, or aggregation $(3,4)$. While E. coli extracts remain a top choice for CFPS, eukaryotic systems have increased in popularity due to growing interest in human and mammalian proteins and their associated post-translational modifications $(5,6)$. In addition, with certain technologies, such as mRNA display and nucleic acid programmable protein arrays (NAPPA), researchers prefer to use eukaryotic expression systems when studying protein-protein interactions (7-9).
Most CFPS systems, such as the ones described below, follow a coupled transcription-translation mechanism in which recombinant DNA is transcribed and translated in situ without purification of the intermediate mRNA strand (Supplementary Figure S1). This approach simplifies the protein synthesis process by minimizing sample handling and reducing the time and cost required to generate a desired protein or set of related proteins. While all major CFPS systems have been analyzed previously $(1,6$, 10), only a limited number of studies have compared the sequence requirements and relative yields in side-by-side assays (11); however, such information would be useful for new users interested in CFPS.

Here we examine the sequence and template preferences of three commonly used commercial eukaryotic CFPS systems: wheat germ extract (WGE) (Promega, Madison, WI), rabbit reticulocyte lysate (RRL) (Promega), and HeLa cell lysate (HCL) (Thermo Scientific, Waltham, MA). For each system, we quantified the amount of luciferase protein generated from circular plasmid and PCR-generated linear DNA containing three different translation enhancing elements in the $5^{\prime}$ untranslated region (UTR) with or without a 62-nucleotide poly-A tail in the $3^{\prime}$ UTR. The $5^{\prime}$ UTR sequences include the 500-nucleotide encephalomyocarditis (EMCV) internal ribosomal entry site (IRES) commonly used to promote cap-independent translation and two minimal translation enhancing elements (TEE) derived from the tobacco mosaic virus (TMV) and the alfalfa mosaic virus (AMV) $(12,13,14)$. Translation reactions were performed in the specified systems, following the manufacturers' recommended protocols, with a template concentration of $40 \mathrm{ng} / \mu \mathrm{L}$ for plasmid DNA and $32 \mathrm{ng} /$ $\mu \mathrm{L}$ for linear DNA templates generated by PCR. Protein expression levels were measured using a Glomax 96-well microplate luminometer (Promega).

Systematic analysis of the $5^{\prime}$ and $3{ }^{\prime}$ UTR sequence determinants for linear and plasmid DNA expressed in WGE, RRL, and $\mathrm{HCL}$ are summarized in Figure 1. Our data illustrate a number of general trends, as well as several lysate-specific properties. In general, we find that (i) plasmid DNA generates $~ 500$-fold more protein than linear DNA, (ii) the 3' poly-A tail plays a minor role in CFPS, and (iii) the most efficient protein translation system is $\mathrm{HCL}$ using plasmids that contain an EMCV IRES in the $5^{\prime}$ UTR. The low efficiency of linear templates has been linked to the degradation of linear DNA in cell lysate (15). The circularization of linear templates can prevent their degradation, increasing yields and is a potential alternative when plasmid DNA is cost-prohibitive for high-throughput assays (16).

Because some biotechnology applications are constrained by a particular lysate system or DNA template design, we also note a number of lysate-specific observations. For WGE, plasmid and linear DNA templates function optimally when AMV

\section{METHOD SUMMARY}

Here we present a comparative analysis of three commonly used eukaryotic cell-free protein synthesis (CFPS) systems. Our results indicate that sequence preferences in the $5^{\prime}$ untranslated region (UTR) of the parent vector have a significant influence on expression yield. This information provides new guidance for designing cell-free protein expression experiments. 
A)

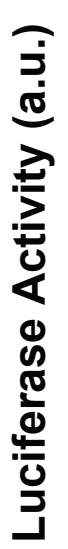

Plasmid

$\square p(A) 62 \square p(A) 0$

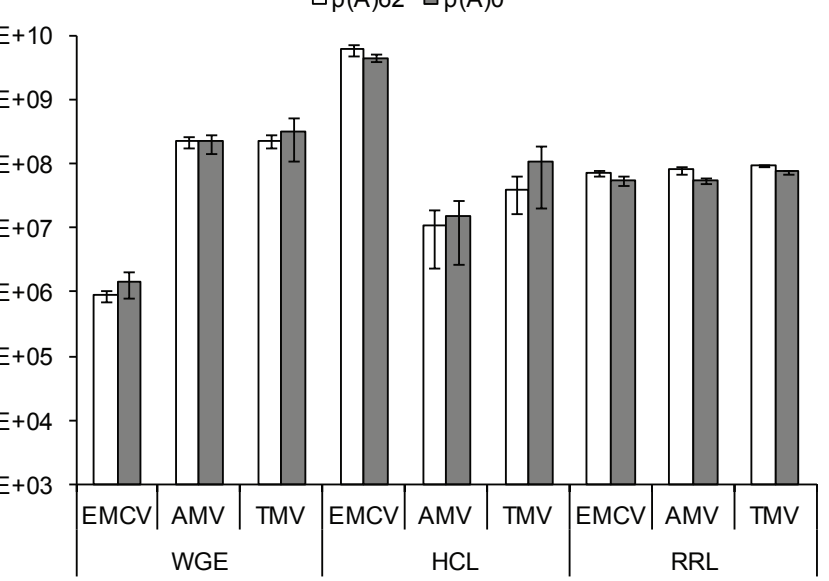

B)

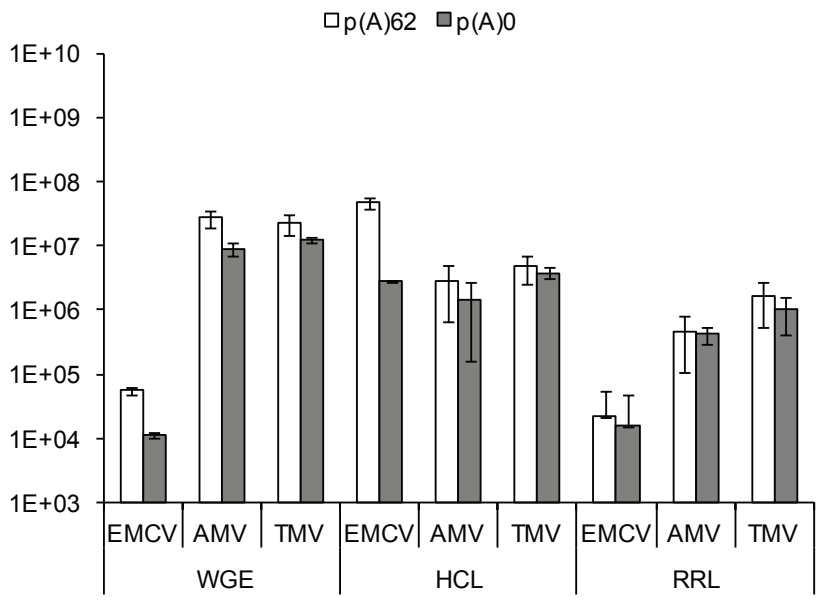

Figure 1. Comparison of luciferase production in cell-free protein synthesis (CFPS) systems. Luciferase constructs engineered with three different translation enhancing elements (EMCV, AMV, or TMV) and the presence (white bars) or absence (gray bars) of a poly-A tail were tested for protein synthesis in three different commercial lysate systems: wheat germ extract (WGE), HeLa cell lysate (HCL), and rabbit reticulocyte lysate $(R R L)$. Values: mean $\pm S D ; n=3$. Luciferase expression was examined with (A) circular plasmids and (B) linear DNA template generated by PCR.

or TMV are present in the 5' UTR. The opposite is true for HCL, which shows a strong preference for the EMCV IRES in the $5^{\prime}$ UTR. Additionally, the translation of linear DNA templates with EMCV is $>10$-fold higher when a poly-A tail is present in the 3' UTR. Interestingly, this was the only example where a poly-A tail made a significant difference in protein synthesis, which stands in contrast to earlier reports where poly-A tails are reported to increase CFPS levels $(17,18)$. The RRL system showed no preference for a $5^{\prime}$ UTR sequence when plasmid DNA was used in the translation reaction; however, linear DNA follows the same trend observed for the WGE system by favoring AMV and TMV in the 5' UTR.

To ensure that our results were general and not specific to luciferase, we constructed five additional plasmids. Each plasmid contained the EMCV IRES in the $5^{\prime}$ UTR, a 3' myc epitope tag and coded for either luciferase or one of four human proteins (TNFRSF21, MYOT, HADHSC, and HRAS) that were randomly chosen to represent a set of proteins with different classifications and sizes. The plasmids were used as templates for CFPS in HCL, WGE, and RRL, and the

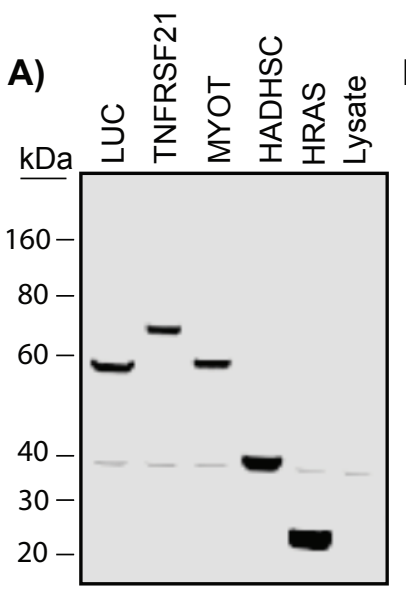

$\mathrm{HCL}$
B)

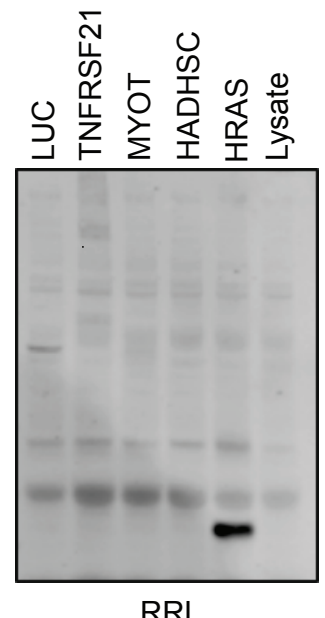

RRL

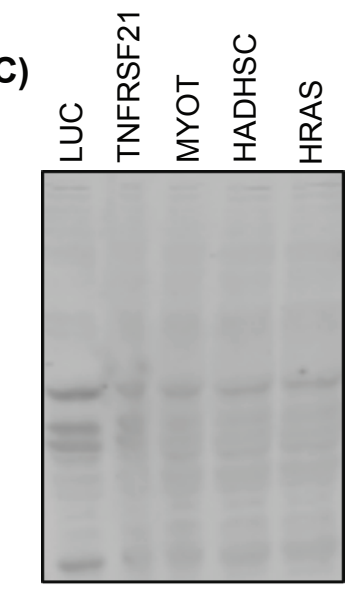

WGE

Figure 2. Western blot analysis of human proteins in HeLa cell lysate (HCL), rabbit reticulocyte lysate (RRL), and wheat germ extract (WGE). Proteins were expressed in (A) HCL, (B) RRL, and (C) WGE commercial cell-free protein synthesis (CFPS) systems from an EMCV-derived plasmid engineered with a C-terminal myc epitope tag and visualized by Western blot with an anti-myc antibody. For the WGE and RRL systems, a luciferase template is provided by the manufacturer as a positive control. While the luciferase used in these Western blots is tagged with c-myc, no significant differences in luciferase activity were observed between the c-myc-tagged and untagged luciferases that were cell-free translated (data not shown), demonstrating that the c-myc tag does not interfere with protein translation and that the c-myc-tagged luciferase is a valid positive control for the Western blots.

resulting proteins were visualized by Western blot using the 9E10-ChIP Grade anti-myc primary antibody (Abcam, Cambridge, UK) and a goat anti-mouse secondary antibody conjugated to an $800 \mathrm{~nm} I \mathrm{R}$ dye (LI-COR Biosciences, Lincoln, Nebraska). Western blot analysis showed strong protein expression in $\mathrm{HCL}$, moderate expression in $R R L$, and undetectable expression in WGE (Figure 2), which is consistent with our previous luciferase results for expression of the EMCV plasmid in HCL. Proteins made in the RRL and WGE systems were visualized upon prolonged exposure (data not shown).

In summary, our results uncover some of the idiosyncrasies of CFPS by identifying optimal DNA templates for commonly used eukaryotic CFPS systems. To maximize translation yields, we recommend using $\mathrm{HCL}$ with commercial plasmids that carry an EMCV IRES in the 5' UTR. Although the cost of $\mathrm{HCL}$ is more than twice that of WGE and RRL on a per reaction basis (Supplementary Table S3), this system is capable of generating 20-50-fold more protein than WGE or RRL. The ability to generate substantially more protein per translation reaction makes $\mathrm{HCL}$ an ideal choice for high-throughput protein expression assays such as NAPPA that require maximal amounts of protein to minimize the occurrence of false negatives. However, because certain technologies such as mRNA display may require linear templates or specific lysate systems, understanding the sequence determinants of all three systems will save time, effort, and cost for researchers interested in generating recombinant protein by CFPS. 


\section{Author contributions}

J.C. conceived the idea for this study. A.L., P.S., and E.H. prepared or provided the materials used in the experiments. E.H., P.S., and A.L. performed the experiments described in the manuscript. A.L., P.S., E.H., and J.C. analyzed and interpreted the data. E.H., A.L., and J.C. wrote the manuscript.

\section{Acknowledgments}

We thank members of the Chaput lab for helpful discussions and comments on the manuscript. This work was supported with funding from the NIH (U54DK093449). This paper is subject to the $\mathrm{NIH}$ Public Access Policy.

\section{Competing interests}

The authors declare no competing interests.

\section{References}

1. Carlson, E.D., R. Gan, C.E. Hodgman, and M.C. Jewett. 2012. Cell-free protein synthesis: applications come of age. Biotechnol. Adv. 30:1185-1194.

2. Murray, C.J. and R. Baliga. 2013. Cell-free translation of peptides and proteins: from high throughput screening to clinical production. Curr. Opin. Chem. Biol. 17:420-426.
3. Katzen, F., G. Chang, and W. Kudlicki. 2005. The past, present and future of cell-free protein synthesis. Trends Biotechnol. 23:150-156.

4. Katzen, F., T.C. Peterson, and W. Kudlicki. 2009. Membrane protein expression: no cells required. Trends Biotechnol. 27:455-460.

5. Endo, Y. and T. Sawasaki. 2006. Cell-free expression systems for eukaryotic protein production. Curr. Opin. Biotechnol. 17:373-380.

6. Saul, J., B. Petritis, S. Sau, F. Rauf, M. Gaskin, B. Ober-Reynolds, I. Mineyev, M. Magee, et al. 2014. Development of a full-length human protein production pipeline. Protein Sci. 23:1123-1135.

7. Roberts, R.W. and J.W. Szostak. 1997. RNA-peptide fusions for the in vitro selection of peptides and proteins. Proc. Natl. Acad. Sci. USA 94:12297-12302.

8. Qiu, J. and J. LaBaer. 2011. Nucleic acid programmable protein array: a just-in-time multiplexed protein expression and purification platform. Methods Enzymol. 500:151-163.

9. He, M., Y. He, Q. Luo, and M. Wang. 2011. From DNA to protein: no living cells required. Process Biochem. 46:615-620.

10. Kobbs, G. 2008. Selecting the cell-free protein expression system that meets your experimental goals. Cell Notes 21:6-9.

11. Mureev, S., O. Lovtun, U.T. Nguyen, and K. Alexandrov. 2009. Species-independent translational leaders facilitate cell-free expression. Nat. Biotechnol. 27:747-752.

12. Elroy-Stein, O., T.R. Fuerst, and B. Moss. 1989. Cap-independent translation of mRNA conferred by encephalomyocarditis virus 5' sequence improves the performance of the vaccinia virus/bacteriophage
T7 hybrid expression system. Proc. Natl. Acad. Sci. USA 86:6126-6130.

13. Gallie, D.R., D.E. Sleat, J.W. Watts, P.C. Turner, and T.M.A. Wilson. 1987. The 5'-leader sequence of tobacco mosaic virus RNA enhances the expression of foreign gene transcripts in vitro and in vivo. Nucleic Acids Res. 15:3257-3273.

14. Jobling, S.A. and L. Gehrke. 1987. Enhanced translation of chimeric messenger RNAs containing a plant viral untranslated leader sequence. Nature 325:622-625.

15. Yang, H.L., L. Ivashkiv, H. Chen, G. Zubay, and M. Cashel. 1980. Cell-free coupled transcriptiontranslation system for investigation of linear DNA segments. Proc. Natl. Acad. Sci. USA 77:7029-7033.

16. Wu, P.S.C., K. Ozawa, S.P. Lim, S.G. Vasudevan, N.E. Dixon, and G. Otting. 2007. Cell-Free Transcription/Translation from PCR-Amplified DNA for High-Throughput NMR Studies. Angew. Chem. Int. Ed. Engl. 46:3356-3358.

17. Bergamini, G., T. Preiss, and M.W. Hentze. 2000. Picornavirus IRESes and the poly(A) tail jointly promote cap-independent translation in a mammalian cell-free system. RNA 6:1781-1790.

18. Jackson, R.J. and N. Standart. 1990. Do the Poly(A) Tail and 3' untranslated region control mRNA translation? Cell 62:15-24.

\section{Received 19 May 2015; accepted 01 July 2015.}

Address correspondence to John C. Chaput, Center for Personalized Medicine and Department of Chemistry and Biochemistry, Arizona State University, Tempe, AZ. E-mail: john.chaput@asu.edu

To purchase reprints of this article, contact biotechniques@fosterprinting.com

\section{MYCOPLASMA CONTROL REAGENTS}

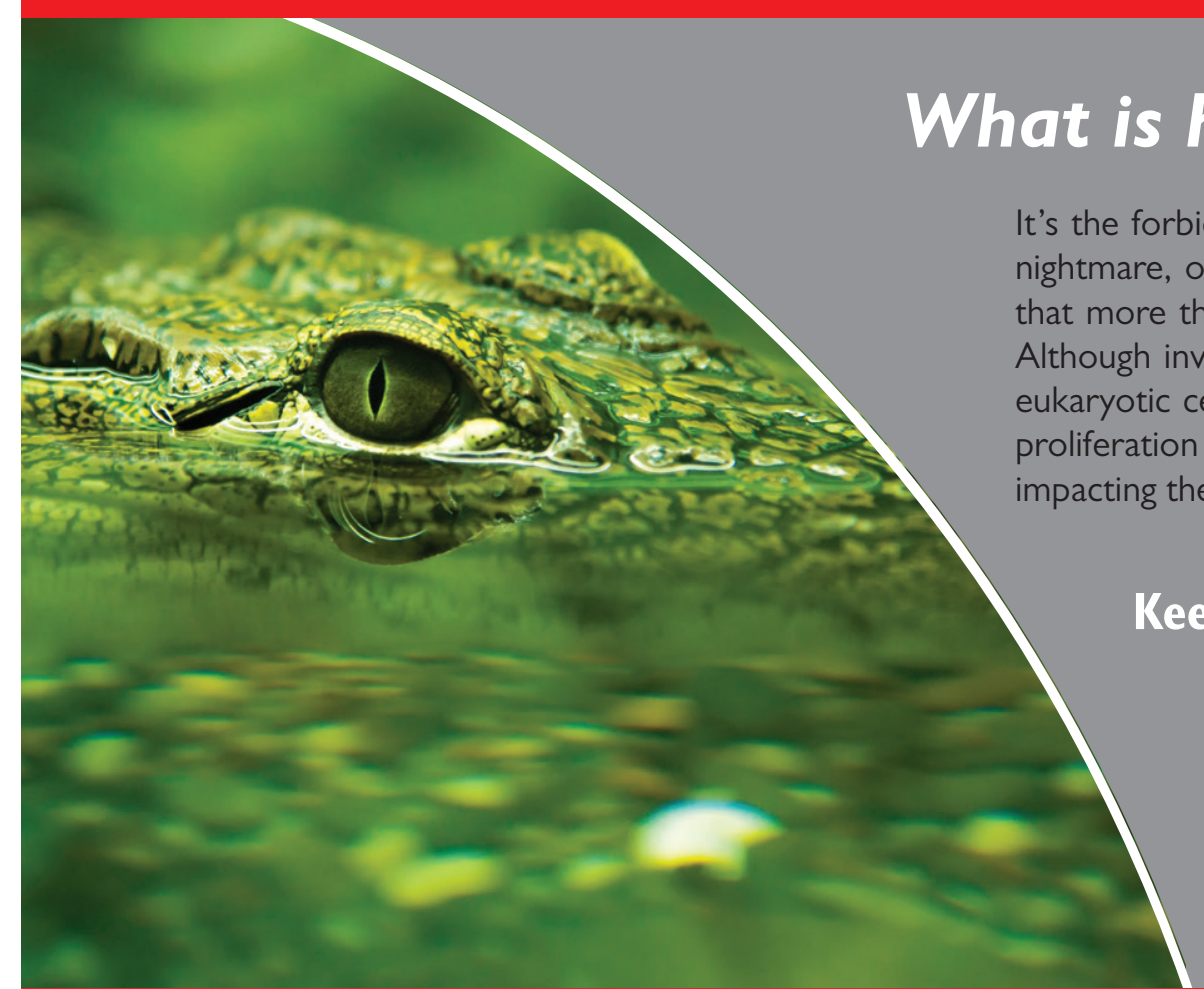

Detect: PlasmoTest ${ }^{\mathrm{TM}}$

Eradicate: Plasmocin ${ }^{\mathrm{TM}}$ - Plasmocure ${ }^{\mathrm{TM}}$

Prevent: Normocin ${ }^{\mathrm{TM}}$ - Normocure ${ }^{\mathrm{TM}}$ 